\title{
Magnetic fingerprints of the very fast jumps of colossal magnetoresistance in the phase-separated manganite $\mathrm{La}_{0.225} \operatorname{Pr}_{0.40} \mathrm{Ca}_{0.375} \mathrm{MnO}_{3}$
}

\author{
F. Macià, ${ }^{1}$ G. Abrill, ${ }^{1}$ A. Hernández-Mínguez, ${ }^{1}$ J. M. Hernandez, ${ }^{1}$ J. Tejada, ${ }^{1}$ and F. Parisi ${ }^{2}$ \\ ${ }^{1}$ Departament de Física Fonamental, Facultat de Física, Universitat de Barcelona, Avinguda Diagonal 647, Planta 4, Edifici nou, \\ 08028 Barcelona, Spain \\ ${ }^{2}$ Departamento de Física, Comisión Nacional de Energía Atómica and Escuela de Ciencia y Tecnología, UNSAM, \\ Avenida Gral Paz 1499 (1650) San Martin, Buenos Aires, Argentina
}

(Received 4 October 2007; revised manuscript received 30 October 2007; published 15 January 2008)

\begin{abstract}
We found that colossal variations of resistance in $\mathrm{La}_{0.225} \mathrm{Pr}_{0.40} \mathrm{Ca}_{0.375} \mathrm{MnO}_{3}$ in about $100 \mu$ s occur simultaneously with magnetic avalanches that last about $1 \mathrm{~ms}$. These findings are interpreted as macroscopic fingerprints of the percolative phase separation upon application of the external magnetic field. Depending on the fraction of the ferromagnetic phase $x$, the colossal magnetoresistance jumps appear to be caused either by fluctuations of $x$, accompanied by fast propagation of the magnetic avalanches through the sample, or by a phase transition from charge ordered antiferromagnetic phase to charge delocalized ferromagnetic phase.

DOI: 10.1103/PhysRevB.77.012403

PACS number(s): 75.10.-b, 45.70.Ht, 75.30.Kz
\end{abstract}

The extraordinary colossal change of resistivity observed in many Mn-based oxides after the application of a magnetic field, colossal magnetoresistance (CMR), is nowadays a very attractive and active field in basic and applied research. ${ }^{1-3} \mathrm{~A}$ well established fact is that these compounds have a very complex phase diagram, characterized by the presence of regions where phases with different magnetic and electronic properties coexist, being the charge delocalized ferromagnetic (CD-FM) and the charge ordered antiferromagnetic (CO-AFM) phases the two most conspicuous ones involved in the phase-separated state. ${ }^{4,5}$

It was argued that a true phase-separated state can develop in systems displaying first order phase transitions (FOPTs) in the presence of quenched disorder. The conjunction between these spread FOPTs with a slow growing dynamic of the equilibrium low temperature phase against the metastable one gives rise to the formation of nonequilibrium "glasslike behaved" states, which are frozen below a state-dependent blocking temperature. ${ }^{6}$ The list of known systems sharing this behavior is quickly increasing: ${ }^{7,8}$ magnetoresistant manganites, ${ }^{5,7,8}$ doped $\mathrm{CeFe}_{2},{ }^{9,10}$ magnetocaloric $\mathrm{Gd}_{5} \mathrm{Ge}_{4},{ }^{11,12}$ etc. The most spectacular feature displayed by these kinds of systems is perhaps the existence of fieldinduced magnetic avalanches (MAs) at low temperatures. Beyond specific characteristics of the magnetic and structural states in each compound, MAs were observed in Co doped $\mathrm{Pr}_{0.5} \mathrm{Ca}_{0.5} \mathrm{MnO}_{3},{ }^{13} \mathrm{Fe}$ doped $\mathrm{La}_{0.5} \mathrm{Ca}_{0.5} \mathrm{MnO}_{3}$ (Ref. 14), $\mathrm{La}_{0.225} \mathrm{Pr}_{0.40} \mathrm{Ca}_{0.375} \mathrm{MnO}_{3},{ }^{15}$ ( $\left.\mathrm{Sm}, \mathrm{Sr}\right) \mathrm{MnO}_{3},{ }^{16,17} \mathrm{CeFe}_{2},{ }^{18}$ and $\mathrm{Gd}_{5} \mathrm{Ge}_{4}$ (Refs. 19-21) among others. One key ingredient for obtaining MA is the existence of strongly blocked metastable states at low temperature. These blocked states remain almost unchanged until the applied magnetic field overcomes certain threshold value. ${ }^{15}$ The very recent report on the spatial propagation of magnetic avalanches ${ }^{22}$ seems to play an important role in the explanation of the abrupt transition between CD-FM and CO-AFM phases. The experimental evidence that this transition occurs via a deflagration phenomenon associated with the fast growth of the size of the CD-FM clusters has introduced fresh ideas in the field. The existence of blocked configurations in the very low tempera- ture regime $(T<6 \mathrm{~K})$ of magnetoresistant manganites opens the possibility to study percolation effects in bulk samples through a fine control of the amount of the conducting phase embedded in the insulating host.

Very interesting percolative models have been proposed, and conclusive microscopic and mesoscopic experiments have been performed. ${ }^{23-25}$ Ultrasharp jumps in the resistivity as a function of temperature in the vicinity of the metalinsulator transition of a $\mathrm{La}_{5 / 8-y} \mathrm{Pr}_{y} \mathrm{Ca}_{3 / 8} \mathrm{MnO}_{3}[\operatorname{LPCM}(y)]$ wire have been recently reported, ${ }^{26}$ showing that percolative transport is enhanced by spatial confinement effects. The formation of filamentary conduction paths was also found in mesoscopic manganite-based devices under the application of magnetic or electric fields. Resistivity jumps observed in such experiments were also tied to the spatial confinement of the current paths. ${ }^{24}$

In this work, we study both the generation of magnetic avalanches and percolation effects in the very low temperature regime of a phase-separated manganite. The control of the conduction channels is here based on physical confinement of the conducting paths, taking advantage of the existence of strongly blocked states. We have monitored phase transition under both the influence of sweeping magnetic fields and the effects induced by surface acoustic waves (SAWs) by simultaneously detecting MAs and resistivity within millisecond scales. Our goal was to elucidate the experimental macroscopic magnetic fingerprints of the percolative transport through the CD-FM domains, which has been suggested to explain the occurrence of the CMR effect.

Then, we proceeded to perform two different kinds of experiments. In both, polycrystalline samples of composition $\mathrm{La}_{0.225} \mathrm{Pr}_{0.40} \mathrm{Ca}_{0.375} \mathrm{MnO}_{3}$ with dimensions of about $1 \times 1 \times 2.5 \mathrm{~mm}^{3}$ were used. The assembly was placed in the bore of a commercial superconducting magnet system capable of producing magnetic fields up to $5 \mathrm{~T}$ and temperatures in the range of 300-2 K. In the first set of experiments, we measured simultaneously the time evolution of the MA associated with the phase transition between the CO-AFM and CD-FM phases and the temperature of the sample. These experiments highlight the heat diffusion process that takes 
(a)

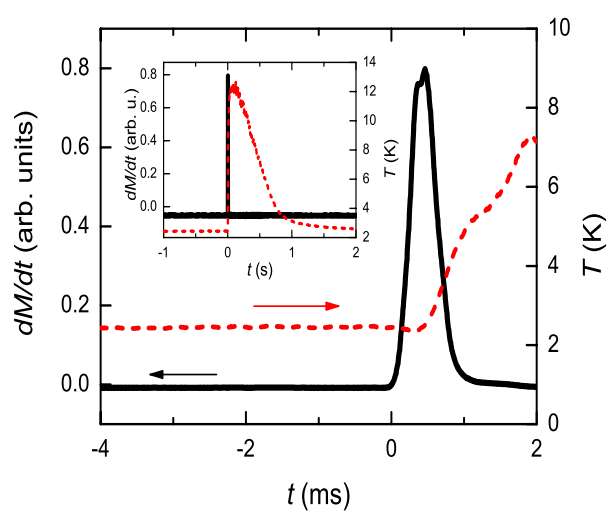

(b)

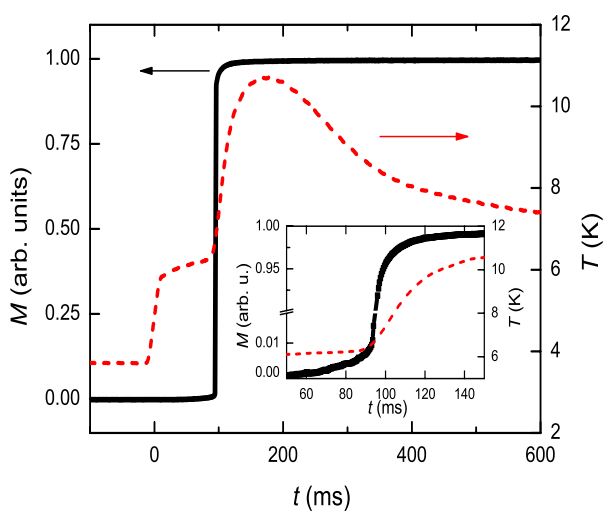

FIG. 1. (Color online) Detection of magnetic avalanches. In (a), time evolution of both the pick-up coil voltage and temperature during a magnetic avalanche, at the same time notice the magnetic field was sweeping at $300 \mathrm{Oe} / \mathrm{s}$. The avalanche occurred spontaneously around $H=30 \mathrm{kOe}$ at $T=2.5 \mathrm{~K}, t=0$ corresponds to the appearance of the avalanches, around $H=30 \mathrm{kOe}$. The inset shows the same graphic in a much spread window. In (b), time evolution of magnetization and temperature during the avalanche process at $T=4 \mathrm{~K}$. The avalanche was ignited by a SAW pulse applied at $t=0$ under a constant applied field, $H=28 \mathrm{kOe}$, in a sample that was cooled under $H=11 \mathrm{kOe}$. The inset shows $M(t)$ and $T(t)$ over a narrower time window.

place during a MA. ${ }^{22}$ We are able to control the ignition of MAs by either sweeping the magnetic field or directly applying surface acoustic waves to the sample.

Figure 1(a) depicts time dependence of the pick-up coil voltage and the temperature variation, while the magnetic field was swept at a constant rate of $300 \mathrm{Oe} / \mathrm{s}$ at the initial temperature $T=2.5 \mathrm{~K}$. The pick-up coil was placed at the center of the sample determining the magnetization variation. A MA was clearly detected by both the pick-up coil and the thermometer at a magnetic field around $H=30 \mathrm{kOe}$. In Fig. 1(b), we show a MA induced at constant magnetic field $H=28 \mathrm{kOe}$ and initial temperature $T=4 \mathrm{~K}$ under the effect of SAWs. In this case, we depict time dependence of magnetization and temperature. The magnetization measurement was carried out by continuously recording the voltage detected by the rf-superconducting quantum interference device of the magnetometer. The SAW pulse was applied at $t=0$ and the MA appeared a few milliseconds later. During that elapsed time where the magnetization hardly changed, the temperature immediately increased after the onset of the SAW pulse and afterwards, the ignition of the MA was accompanied by a second, more pronounced, increase in temperature. MAs occur in times of a few milliseconds and the remarkable point is that no matter what was the initial value of the CD-FM phase $x$ of the sample, MAs are always accompanied by a rapid change in the temperature of the sample. We can thus monitor the occurrence of the magnetic avalanches by just reading the temperature variation of the thermometer over the sample. Slow magnetization variations of the sample do not produce any appreciable increase on its temperature. $^{22}$

In the next, we discuss the second type of experiments in which we directly detect the very fast jumps in the resistance associated with the percolative phase separation induced by the external magnetic field. In these experiments, in addition to measuring the temperature variation of the sample, we connected two wires at the two edges of the sample and injected a small current $(10 \mu \mathrm{A})$ to measure the voltage drop across the sample. We first performed the field cooling process of the sample at a constant cooling rate down to the lowest desired temperature between 2 and $4 \mathrm{~K}$. This process allows us to control the concentration $x$ of the CD-FM phase. The concentration $x$ is fully determined by the cooling rate and the value of the external applied magnetic field during the cooling process. In the case of the zero field cooling process, the $x$ value was lower than $7 \%$ for temperatures between 2 and $4 \mathrm{~K}$, indicating that the sample was mostly in the CO-AFM phase. The magnetization after MA always reach the value corresponding to the saturation magnetization of the ferromagnetic phase, $x=1$. It has been recently shown that the magnetic field values at which the avalanches do appear are fully determined by the value of $x .^{22}$ Two main aspects should be noticed here. The first one highlights the fact that the micrometer size of the CD-FM domains seems to be a good reason to explain the deterministic behavior of the appearance of the magnetic avalanches in a sample of a few millimeter. ${ }^{3}$ The second aspect refers to the percolative phase separation needed to explain the electrical conductor behavior of randomly connected CD-FM clusters. This problem is similar to the case of having an electrical network and calculating the probability $p$, which all nodes of the network are connected with an electrical current flowing through them. When the network has a large number of nodes, the probability $p$ becomes critical; below a threshold value of $p$, the probability of percolation is always 0 and above that value is always 1 . In our case, $p$ is related to the concentration and patterning of the two phases in the sample. From the measurement of the electrical resistance of the sample for different values of $x$ at a zero magnetic field and temperatures between 2 and $4 \mathrm{~K}$, we clearly stated that for $x<7 \%$, the sample was a perfect insulator with a resistance of gigaohms, while for $x>10 \%$, the electrical resistance decreased to hundreds of kilohms. Resistances of gigaohms overflow the measuring limits of our voltmeters, and consequently, we consider the manganite as an insulator; open circuit (OC). On the other hand, the sample having $x=1$ corresponds to the 


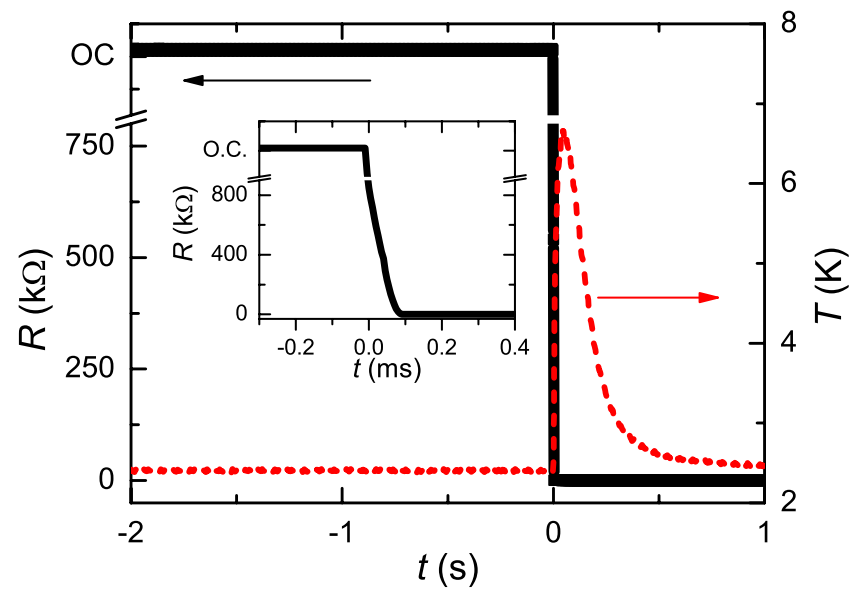

FIG. 2. (Color online) Temperature and electrical resistance evolution during a MA. The sample was zero field cooled until $T$ $=2.5 \mathrm{~K}$. The magnetic field was swept up to $5 \mathrm{~T}$ with a rate of $300 \mathrm{Oe} / \mathrm{s}$. Near $H=30 \mathrm{kOe}$ (i.e., $t=0$ ), the MA is clearly detected with the thermometer at the same time as the resistivity abruptly changes from insulator to a few hundreds of ohms. The inset corresponds to a magnification of the resistance evolution during the CMR avalanche. Zero time is considered at the appearance of the avalanche.

case having only CD-FM phase, with electrical resistance of a few hundreds of ohms.

Figure 2 shows the evolution, after cooling the sample in zero external magnetic field until $T=2.5 \mathrm{~K}$, being $x=6 \%$, of both the electrical resistance and the temperature of the sample when the magnetic field is swept from 0 up to $4 \mathrm{~T}$. From this figure, we deduce that the electrical resistance does not change until the magnetic field reaches the threshold value at which the MA takes place. The MA was accompanied by an abrupt and fast reduction in the electrical resistance. The inset shows a magnification of the CMR avalanche around the instant at which MA took place. The abrupt resistance jump always occurs in times of the order of $100 \mu$ s and goes from insulating values to a few hundreds of ohms.

Figure 3 shows the evolutions of both electrical resistance and temperature for the same case as those reported in Fig. 2 but for a temperature of $3 \mathrm{~K}$. While the initial state at $3 \mathrm{~K}$ and $H=0$ was the same as that at $2.5 \mathrm{~K}$, the evolution to a fully ferromagnetic phase is different when the magnetic field is swept. A few seconds before the occurrence of the MA, the sample abruptly decreased from OC to hundreds of kilohms. After this initial CMR effect, the resistivity slowly decreased until the MA took place and then, a small, but very fast, change in the resistance was detected. It can also be seen in Fig. 3(b), magnification of Fig. 3(a), that the resistance evolved in time (e.g. sweeping the magnetic field) following a sequence of different abrupt and fast changes instead of a monotonously decreasing downward trend.

It is well known that when either the sweeping fields are slow or the fraction of CD-FM phase is larger than some critical value $x$ (this value is deterministic and depends on the size and geometry of the sample ${ }^{22}$ ), MAs do not occur and the CD-FM phase follows a continuous evolution until (a)

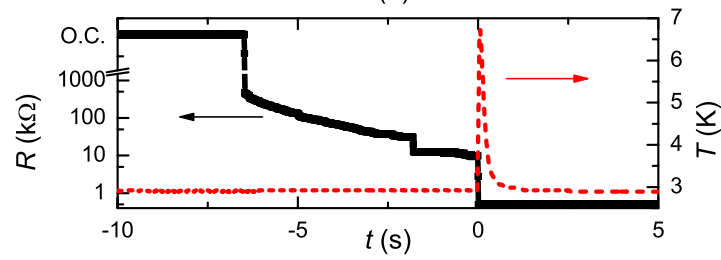

(b)

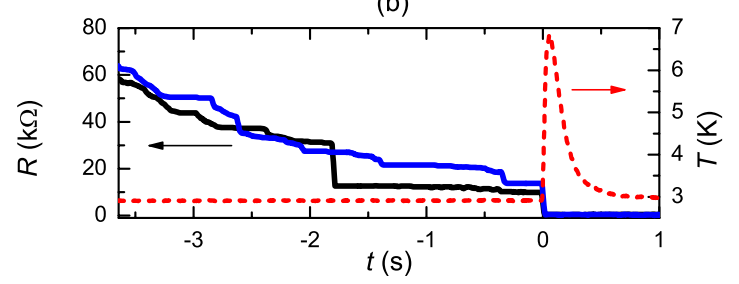

FIG. 3. (Color online) Temperature and electrical resistance evolution during a MA with earlier percolation. In (a), the sample was zero field cooled until $T=3 \mathrm{~K}$. The magnetic field was swept up to $5 \mathrm{~T}$ with a rate of $300 \mathrm{Oe} / \mathrm{s}$. The MA is clearly detected with the thermometer near $H=30 \mathrm{kOe}$ (i.e., $t=0$ ) and at the same time a small and sudden resistivity change appeared. Before the occurrence of the MA, the resistance abruptly decreased from $\mathrm{OC}$ to hundreds of kilohms and then other small and fast changes are detected. Zero time is considered at the appearance of the avalanche. In (b), a magnification of (a). Two curves of the same experiment are plotted in order to elucidate the stochasticity of the resistance and the deterministic character of the MA.

the whole sample becomes ferromagnetic. Figure 4 shows the evolution of electrical resistance in one of these cases. The sample was field cooled with a magnetic field $H$ $=12 \mathrm{kOe}$, and the value of $x$ when the lowest temperature $(T=2.9 \mathrm{~K})$ was reached was approximately $25 \%$. The electrical resistance of the sample in this case was $300 \mathrm{k} \Omega$. We can also observe in the figure that the temperature of the sample remained constant with the sweeping magnetic field indicating the absence of MA. In this case, despite the slow and continuous evolution of the magnetization due to relaxation, the electrical resistance had abrupt changes in times of the order of hundreds of microseconds, whereas in these time scales, the sweeping magnetic field did hardly modify the

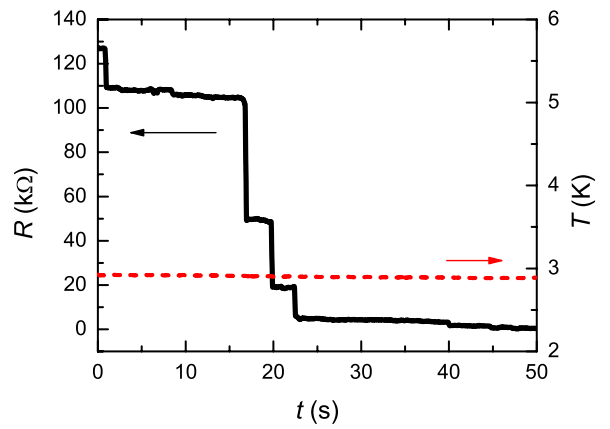

FIG. 4. (Color online) Temperature and electrical resistance evolution without MA. The sample was field cooled with $H$ $=12 \mathrm{kOe}$ until $T=2.9 \mathrm{~K}$. There is no MA in this case, but several resistance jumps can be observed. Time origin $(t=0)$ has been considered arbitrarily around $H=15 \mathrm{kOe}$. Sweep field of $300 \mathrm{Oe} / \mathrm{s}$. 
value of $x$. These abrupt and fast resistance variations are only due to the patterning connecting the CD-FM clusters in the sample. This is the experimental evidence that the percolative phase separation induced by the effect of the external magnetic field is fully responsible for the CMR jumps. Due to the proximity in the energies of the coexisting phases, it is very easy to displace the phase boundaries by, for example, an external magnetic field. This possibility is at the basis of the colossal magnetoresistance effect: small variations of the amount of the coexisting phases can lead to the percolation of the conducting phase (CD-FM), giving raise to huge changes in the resistivity.

In conclusion, our experiments clearly show that MAs do always induce fast resistance jumps. These jumps may also occur with no appreciable changes in the magnetization of the sample. We may, therefore, link MA induced either by the external magnetic field or by SAWs associated with the phase transition from CO-AFM to CD-FM phases with co- lossal changes of the resistance. As a matter of fact, for $x$ values smaller than $7 \%$, the fast resistance jumps are always due to the occurrence of MA at well determined values of the magnetic field and a certain power of the SAWs. For $x$ values larger than $10 \%$, the resistance jumps are mostly due to the percolation effect associated with the rearranging of the CD-FM clusters inside the sample.

This work was supported by Contract No. MAT200506162 from the Spanish Ministerio de Educación y Ciencia (MEyC). A.H.-M. and F.M. thank the MEyC for a research grant. J.M.H. thanks the MEyC and the Universitat de Barcelona for a Ramón y Cajal research contract. The authors also thank Gabriela Leyva for preparing the sample. We thank P. V. Santos, W. Seidel, and S. Krauss for the fabrication of the piezoelements and the Acciones Integradas (Spain)/DAAD (Germany) program for financial support.
${ }^{1}$ S. Jin, T. H. Tiefel, M. McCormack, R. A. Fastnacht, R. Ramesh, and L. H. Chen, Science 264, 413 (1994).

${ }^{2}$ F. Parisi, P. Levy, L. Ghivelder, G. Polla, and D. Vega, Phys. Rev. B 63, 144419 (2001).

${ }^{3}$ R. von Helmolt, J. Wecker, B. Holzapfel, L. Schultz, and K. Samwer, Phys. Rev. Lett. 71, 2331 (1993).

${ }^{4}$ A. Moreo, S. Yunoki, and E. Dagotto, Science 283, 2034 (1999).

${ }^{5}$ J. Sacanell, F. Parisi, J. C. P. Campoy, and L. Ghivelder, Phys. Rev. B 73, 014403 (2006).

${ }^{6}$ Y. Imry and M. Wortis, Phys. Rev. B 19, 3580 (1979).

${ }^{7}$ L. Ghivelder and F. Parisi, Phys. Rev. B 71, 184425 (2005).

${ }^{8}$ P. A. Sharma, S. B. Kim, T. Y. Koo, S. Guha, and S.-W. Cheong, Phys. Rev. B 71, 224416 (2005).

${ }^{9}$ M. K. Chattopadhyay, S. B. Roy, and P. Chaddah, Phys. Rev. B 72, 180401(R) (2005).

${ }^{10}$ K. Kumar, A. K. Pramanik, A. Banerjee, P. Chaddah, S. B. Roy, S. Park, C. L. Zhang, and S.-W. Cheong, Phys. Rev. B 73, 184435 (2006).

${ }^{11}$ S. B. Roy, M. K. Chattopadhyay, A. Banjerjee, P. Chaddah, J. D. Moore, G. K. Perkins, L. F. Cohen, K. A. Gschneidner, Jr., and V. K. Pecharsky, Phys. Rev. B 75, 184410 (2007).

${ }^{12}$ S. B. Roy, M. K. Chattopadhyay, P. Chaddah, J. D. Moore, G. K. Perkins, L. F. Cohen, K. A. Gschneidner, Jr., and V. K. Pecharsky, Phys. Rev. B 74, 012403 (2006).

${ }^{13}$ R. Mahendiran, A. Maignan, S. Hébert, C. Martin, M. Hervieu, B. Raveau, J. F. Mitchell, and P. Schiffer, Phys. Rev. Lett. 89, 286602 (2002).

${ }^{14}$ L. Granja, R. S. Freitas, L. Ghivelder, G. Polla, F. Parisi, and P.
Levy, J. Low Temp. Phys. 135, 111 (2004).

${ }^{15}$ L. Ghivelder, R. S. Freitas, M. G. das Virgens, M. A. Continenino, H. Martinho, L. Granja, M. Quintero, G. Leyva, P. Levy, and F. Parisi, Phys. Rev. B 69, 214414 (2004).

${ }^{16}$ L. M. Fisher, A. V. Kalinov, I. F. Voloshin, N. A. Babushkina, D. I. Khomskii, Y. Zhang, and T. T. M. Palstra, Phys. Rev. B 70, 212411 (2004).

${ }^{17}$ A. V. Kalinov, L. Fisher, I. Voloshin, N. Babushkina, D. Khomskii, and T. Palstra, J. Magn. Magn. Mater. 300, e399 (2006).

${ }^{18}$ S. B. Roy, M. K. Chattopadhyay, P. Chaddah, and A. K. Nigam, Phys. Rev. B 71, 174413 (2005).

${ }^{19}$ V. Hardy, S. Majumdar, S. J. Crowe, M. R. Lees, D. M. Paul, L. Hervé, A. Maignan, S. Hébert, C. Martin, C. Yaicle, M. Hervieu, and B. Raveau, Phys. Rev. B 69, 020407(R) (2004).

${ }^{20}$ E. M. Levin, V. K. Pecharsky, K. A. Gschneidner, Jr., and G. J. Miller, Phys. Rev. B 64, 235103 (2001).

${ }^{21}$ H. Tang, V. K. Pecharsky, K. A. Gschneidner, Jr., and A. O. Pecharsky, Phys. Rev. B 69, 064410 (2004).

${ }^{22}$ F. Macià, A. Hernández-Mínguez, G. Abril, J. M. Hernandez, A. García-Santiago, J. Tejada, F. Parisi, and P. V. Santos, Phys. Rev. B 76, 174424 (2007).

${ }^{23}$ M. Uehara, S. Mori, C. H. Chen, and S.-W. Cheong, Nature (London) 399, 560 (1999).

${ }^{24}$ T. Wu and J. F. Mitchell, Phys. Rev. B 74, 214423 (2006).

${ }^{25}$ L. Zhang, C. Israel, A. Biswas, R. L. Greene, and A. de Lozanne, Science 298, 805 (2003).

${ }^{26}$ H. Y. Zhai, J. X. Ma, D. T. Gillaspie, X. G. Zhang, T. Z. Ward, E. W. Plummer, and J. Shen, Phys. Rev. Lett. 97, 167201 (2006). 